policy makers and gives insight into the requirements of how to realise them. A report will be published end of the year 2017.

Discussion The study has identified gaps concerning the performance of empowerment and participation in the area of vocational education and training. There is a need for innovative learning environments especially for young workers and professionals to improve their health-competence.

\section{OCCUPATIONAL PSYCHOLOGY AND THERAPEUTIC YOGA -TOOLS FOR CRACKING HSE INTERNATIONAL CERTIFICATION EXAMS}

M Jayandran*. Green World Group Training and Consulting LLC, Chennai, India

\subsection{6/oemed-2018-ICOHabstracts.289}

Introduction Occupational Psychology is a Science of measuring psychological variables, such as knowledge, Self esteem, skills and abilities of an employee Understanding human behaviour in the workplace with respect to organisations principle and policies. A field that is concerned with the safety, physical and mental well being of an employee. Therapeutic Yoga - is an initiative which can be implemented by the Employer appointed competent Occupational yoga therapist and can be followed by the worker includes breath control, simple meditation, adoption of a specific bodily postures practiced for health and relaxation so as to perform well in the assigned tasks especially in high risk activities. It can be applied to maintain worker fitness in high risk activities like Work at Height and Confined Space and to remove their respective phobias i.e., Acrophobia and Claustrophobia and Mnemophobia for the aspirants who are undertaking International HSE competitive exams like NEBSOH, CSP etc.,. The Employee well being is a very important factor to control accidents in the workplace. Individual behaviour like Attitude, Competence, Perception, will play a vital role in Work Place Safety. This Individual behaviour and the mental well being can be highly improved if the employer applies the idea of Occupational Psychology and Therapeutic yoga.ILO 161 and ILO 1959 R112 defines 'occupational health services' states that safe and healthy working environment which will facilitate optimal physical and mental health in relation to work and the adaptation of work to the capabilities of workers Methods

a. Counselling Therapy: Counselling aims to help individual/

Employees to manage their accident prone psychological

difficulties and converting them to contribute for

Organisation Safety Occupational Psychologist has to

understand the types of psychological issues exist in the workplace and has to decide the counselling method as follows, Psychoanalytic therapy or talk therapy can be done by listening to Employees talk about their lives will release their personal stress.

b. Therapeutic Yoga blends postures, breath work, hands-on healing, meditation techniques which will improve the memory level for the aspirants who undergoing HSE training and preparing for competitive international HSE certification exams.

Results By applying counselling therapies and meditation stress control methods it has observed that the class test performance and the confidence level have improved very positively. Out of 6 aspirants, $60 \%$ improvement in terms of confidence and performance has achieved.
Conclusion The human mind and the individual factors are very challenging for the HSE Professionals. Continuous HSE trainings and knowledge updating is must for Safety Professionals. In between their work commitment, aspirants are emphasised by the organisation to take international HSE Certifications like IDIP NEBOSH, NEBOSH IGC, CSP. The aspirants greatly affected by mental stress with Mnemophobia. By adopting counselling therapies and therapeutic yoga to my aspirants, I found lot of improvement in their confidence as well as performance level. I recommend this method can be applied for other high risk activities like work at height, confined space to improve the physical and mental fitness of the workers.

Discussions Counselling aims to help individual/Employees to manage their accident prone psychological difficulties, builds confidence and converting them to contribute for Organisation Safety. Occupational Psychologist has to understand the types of psychological issues exist in the workplace and has to decide the counselling method as follows, Psychoanalytic therapy or talk therapy can be done by listening to Employees talk about their lives will release their personal stress. In Behavioural therapy, employees encouraged to gradually face their fear of heights by explaining about the safety precautions adapted in Work at heights. Group therapy is a form of psychotherapy where two or more employees will insist to play a game with their superiors will pave the way healthy dignified relationship. Art therapy involves the use of artistic means to work through difficult emotions. It helps individuals who are having trouble discussing their problems verbally. in the same way, therapeutic yoga postures will boost the mental as well as, physical fitness.

Being a HSE Tutor handling HSE Professionals of different industrial background and tutoring them to take up international HSE certifications like IOSH MS, NEBOSH IGC etc., I identified they are undergoing severe stress and psychological issues due to so many reasons. So, I applied this concept of Counselling therapies, therapeutic yoga and found improvement as follows: Absenteeism rate reduced, Motivation and self confidence increased, Considerable amount of optimistic changes observed in Daily test performance. I suggest, the safety professionals has to implement this techniques to improve the resilience, fitness of the employees so as to create good safety culture and competent work group for high hazardous activities like work at height, confined space safety.

\section{THE EFFECTS OF TRAINING MANAGERS ON MANAGEMENT COMPETENCIES ON WORK ENGAGEMENT OF THEIR SUBORDINATES: A SINGLE- GROUP PRE- AND POST-TEST STUDY}

Y Sekiya*, H Adachi, K Imamura, K Watanabe, N Kawakami. Department of Mental Health, Graduate School of Medicine, The University of Tokyo, Tokyo, Japan

\subsection{6/oemed-2018-ICOHabstracts.290}

Introduction The UK Health and Safety Executive (HSE) developed the concept and measure called 'Stress management competency indicator tool' of management competencies to prevent and reduce stress at work, and has shown some beneficial effect of training managers for these competencies (Yarker, et al, 2008). The purpose of this study was to examine the effect of a training program for managers developed 